\title{
Síntesis de arcilla pilareada con titanio (Ti-PILC) y transesterificación de aceite de ricino
}

\section{Synthesis of titanium pillared clay (Ti-PILC) and castor oil transesterification}

Presentación: 07/10/2020

\section{Doctorando:}

\section{Elias Enrique Origlia}

Centro de Investigación y Tecnología Química (CITeQ) Universidad Tecnológica Nacional (UTN) Consejo Nacional de Investigaciones Científicas y Técnicas (CONICET), Maestro López esq. Cruz Roja Argentina, Córdoba, Argentina

eliasoriglia@gmail.com

\section{Director/a:}

\section{Sandra Graciela Casuscelli}

\section{Co-director/a:}

\section{Analía Laura Cánepa}

\section{Resumen}

En el presente estudio se espera difundir a la comunidad científica los resultados obtenidos, tanto en la síntesis de arcillas pilareadas de titanio (Ti-PILC) como en la transesterificación de aceite de ricino. Ambos procedimientos forman parte de los avances de investigación en la tesis doctoral denominada "Síntesis de materiales como catalizadores para la epoxidación de ésteres metílicos de ácidos grasos (FAME's) obtenidos de aceites vegetales no comestibles". Debido al escaso tiempo, solo se expondrán algunos resultados de la síntesis del catalizador heterogéneo (Ti-PILC), como así también la transesterificación del aceite de ricino para obtener la mezcla de FAME's, materia prima necesaria para la epoxidación propuesta en el tema de tesis original. Los FAME's obtenidos a partir del aceite de ricino se caracterizaron por Espectroscopia Infrarroja con Transformada de Fourier (FTIR). Las arcillas se caracterizaron por isotermas de adsorción-desorción de $\mathrm{N}_{2}$. El material pilareado presentó área específica superior respecto a la arcilla original (Na-mont).

Como próximo objetivo se evaluará el material catalítico en las reacciones de epoxidación. Además, se procederá a identificar los productos de reacción por diferentes técnicas analíticas, tales como: Cromatografía gaseosa (GC), Espectrometría de masa acoplada a cromatografía gaseosa (MS-GC), FTIR y Resonancia magnética nuclear de protones $\left({ }^{1} \mathrm{HRMN}\right)$. Por último, se buscará correlacionar las propiedades fisicoquímicas de los materiales con su actividad catalítica, a fin de rediseñar, si es necesario, los catalizadores que hagan posible optimizar la obtención de los productos buscados (epóxidos).

Palabras clave: Arcilla Pilareada de Titanio, Ésteres metílicos de ácidos grasos, Aceite de Ricino, Transesterificación

\begin{abstract}
In the present study, it is expected to disseminate to the scientific community the results obtained in the synthesis of titanium pillared clays (Ti-PILC) and in the transesterification of castor oil. Both process are part of the research advances in the doctoral
\end{abstract}


thesis called "Synthesis of materials as catalysts for the epoxidation of fatty acid methyl esters (FAME's) derived from inedible vegetable oils". Due to the short time, only some results of the synthesis of the heterogeneous catalyst (Ti-PILC) will be exposed, as well as the transesterification of castor oil to obtain the mixture of FAME's, raw material necessary for the proposed epoxidation in the original thesis topic. FAME's obtained from castor oil are characterized by Fourier Transformed Infrared Spectroscopy (FTIR). The clays were characterized by adsorption-desorption isotherms of N2. The piled material presented a higher specific area than the original clay (Na-mont).

As the next objective, the catalytic material will be evaluated, in epoxidation reactions. In addition, the reaction products will be identified by different analytical techniques, such as: Gas chromatography (GC), Mass spectrometry coupled to gas chromatography (MS-GC), FTIR and Proton nuclear magnetic resonance (1HRMN). Finally, we will seek to correlate the physicochemical properties of the materials with their catalytic activity, in order to redesign, if it is necessary, the catalysts that make it possible to optimize obtaining of products sought (epoxides).

Keywords: Titanium pillared clays, Fatty Acids of Methyl Esters, Castor Oil, Transesterification

\section{Introducción}

Uno de los principales desafíos de la química contemporánea es el desarrollo de tecnologías alternativas que ejerzan una menor presión sobre el entorno natural, es decir, que sean más limpias, más seguras y respetuosas con el medio ambiente. Para llevar adelante estos objetivos, resulta útil la utilización de catalizadores heterogéneos. Entre los materiales atractivos para ser empleados como catalizadores surgen las PILC's (arcillas pilareadas), las cuales son materiales micro y mesoporos que poseen una buena distribución de poros, alta área específica y estabilidad térmica, por lo tanto, tienen una amplia gama de aplicaciones potenciales en procesos catalíticos [1]. Además, las arcillas son abundantes en la naturaleza y tienen bajo costo, encontrándose importantes yacimientos en las provincias de Córdoba, San Juan y Neuquén.

Las PILC's pueden prepararse en base a las conocidas propiedades de intercambio iónico y de hinchamiento que poseen las arcillas naturales. Estas propiedades permiten el intercambio de los cationes presentes en la arcilla natural por polioxocationes de alto peso molecular, producto de la hidrólisis de ciertos metales. Tras calcinación, estos polihidroxicationes se descomponen en los correspondientes óxidos metálicos que actúan como pilares de las láminas de la arcilla. Así, se obtienen las denominadas PILC's. Hasta el momento se han reportado diferentes especies policatiónicas como precursoras de los pilares de la arcilla, los cuales contienen diferentes metales, tales como: Al, Fe, Ti, V, Ce y Zr. En el presente trabajo se sintetizó una arcilla pilareada con Ti (Ti-PILC).

La producción de energía y productos químicos a partir de recursos sustentables ha promovido que la valorización de la biomasa se convierta en una importante área de investigación [2]. En este sentido, la biomasa ofrece una alternativa ideal a los recursos fósiles y ha sido propuesta como una fuente de carbón "verde" equivalente al petróleo. Entre las diferentes fuentes de biomasa se encuentran los aceites vegetales no comestibles, como por ejemplo el aceite de ricino, el cual se obtiene de la semilla de tártago y no tiene uso agrícola o ganadero actual debido a que es tóxico para su consumo. El aceite de ricino o aceite de castor presenta gran interés ya que puede emplearse como materia prima para producir energía y productos químicos sin competir con la producción de alimentos. El tártago que hasta hace algunas décadas solía ser considerado plaga, produce semillas con buena cantidad de aceite de ricino, entre el 40 y $55 \%$ en peso y se caracteriza por su elevado contenido de ácido ricinoleico $(\sim 85-90 \%)$, el cual contiene una insaturación en el carbono 9 y un grupo hidroxilo en el carbono 12, como podemos observar en la Figura 1. La aparición del doble enlace y el grupo hidroxilo en el ácido ricinoleico hace que el aceite de ricino sea

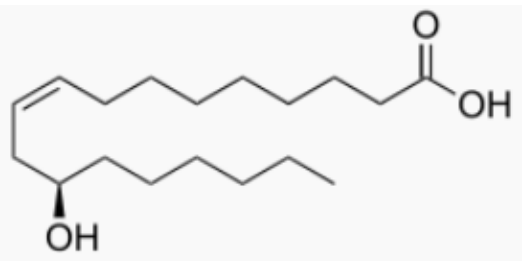

Figura 1. Estructura del ác. ricinoleico 
químicamente muy diferente de los otros aceites; especialmente su elevada viscosidad y polaridad lo hacen extremadamente valioso para la producción industrial de recubrimientos, plásticos y cosméticos, etc. [3].

La importancia del aceite de ricino como materia prima para la industria química se debe a que los triglicéridos, sus principales constituyentes, pueden funcionalizarse a través de diferentes reacciones químicas [4]. Así, la oleoquímica es la rama de la química que se ocupa del estudio de la funcionalización de los aceites y grasas naturales para producir sustancias de alto valor agregado como ésteres, alcoholes, epóxidos, sales grasas, aminas grasas etc., las cuales se emplean en la elaboración de diferentes productos. Otra ventaja de usar ricino es que se puede cultivar en tierras semiáridas que requieren menos mantenimiento, menos fertilidad del suelo y menos agua en comparación con las tierras usadas para el cultivo de aceites vegetales comestibles [5].

Para epoxidar el aceite de ricino, previamente se debe realizar la transesterificación de sus triglicéridos para obtener la mezcla de FAME's (biodiesel). La transesterificación generalmente se lleva a cabo utilizando alcoholes primarios y secundarios [6]. El metanol es el alcohol más usado porque es el menos costoso y muestra ventajas químicas como su cadena más corta y su naturaleza polar. Además, no forma azeótropo, por lo que es más fácil de recuperar que el etanol [7]. La transesterificación se puede llevar a cabo utilizando ácidos (heterogéneos), álcalis (homogéneos) o enzimas como catalizadores de la reacción.

En bibliografía se encuentran trabajos de transesterificación de aceite de ricino a través de catalizadores homogéneos. Elango y col. [8] optimizaron el proceso de transesterificación para producir biodiesel, utilizando 1,25\% en peso de $\mathrm{KOH}$, relación molar metanol: aceite de $12: 1$ a $60{ }^{\circ} \mathrm{C}$ por 60 minutos, obteniendo 94,9\% de conversión de FAME. Keera y col. [9] lograron una conversión de $95 \%$ utilizando $1 \%$ en peso de $\mathrm{KOH}, 9: 1$ de relación molar metanol: aceite a $60{ }^{\circ} \mathrm{C}$ por 30 minutos. En el presente estudio se utilizó la reacción de transesterificación con metanol (metanólisis) en una catálisis homogénea con $\mathrm{KOH}$, como podemos observar en la Figura 2:

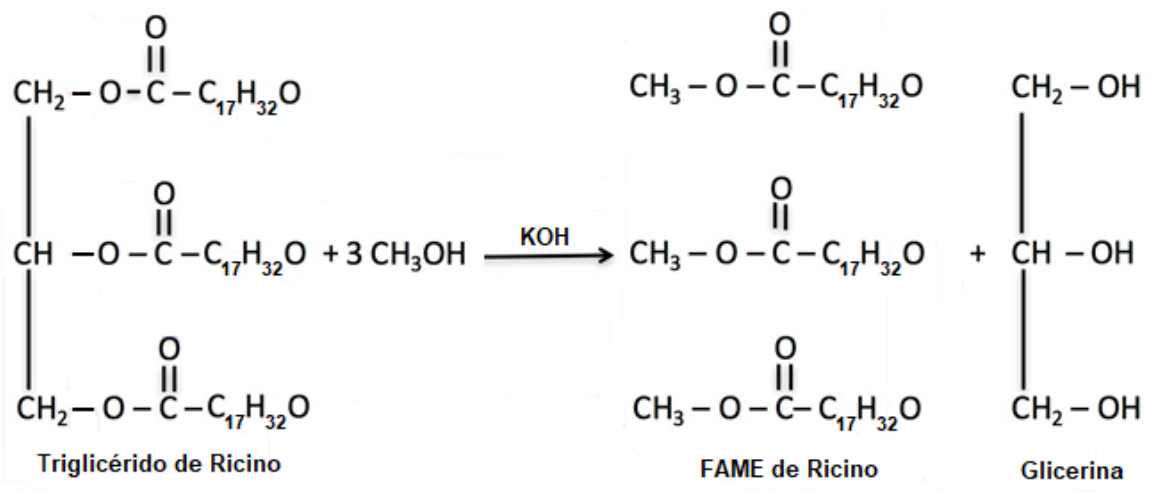

Figura 2. Reacción de Transesterificación de Ricino con metanol y KOH como catalizador.

\section{Metodología}

La PILC se sintetizó a partir de una arcilla natural del tipo montmorillonita (mont) proveniente de la Provincia de San Juan. El primer paso consistió en purificar y obtener la arcilla intercambiada con sodio (Na-mont), para lo cual se pesó $20 \mathrm{~g}$ de arcilla natural y se procedió a tamizarla en un tamiz de malla 200 para eliminar impurezas de gran tamaño. Luego se la colocó dentro de un matraz Erlenmeyer con agua destilada y se procedió a agitar la mezcla por 30 minutos para homogenizarla. Seguidamente se dejó reposar dicha mezcla durante $12 \mathrm{~h}$ para separar y eliminar el cuarzo ubicado en el fondo de una probeta. El sobrenadante se colocó en un balón de 1L y se puso en contacto con una solución de $300 \mathrm{~mL}$ de $\mathrm{NaCl} 1 \mathrm{M}$ (la cual se agregó gota a gota) a temperatura ambiente con agitación mecánica. La mezcla se mantuvo agitando durante $24 \mathrm{~h}$ para lograr un mayor contacto e intercambiar la mayor cantidad de iones que posee la arcilla $\left(\mathrm{Ca}^{2+}, \mathrm{Mg}^{2+}\right.$, etc.) por iones $\mathrm{Na}^{+}$. La fracción sólida se separó por centrifugación, se lavó varias veces hasta eliminar los iones $\mathrm{Cl}^{-}$y se dejó secando a temperatura ambiente. El siguiente paso fue sintetizar la Ti-PILC a partir de la Na-mont obtenida anteriormente. La solución pilareante se preparó agregando gota a gota 2,5 
$\mathrm{mL}$ de Butóxido de titanio (99,9\%, Aldrich) a una solución de 3,3 mL de $\mathrm{HCl}$ (6 M) con agitación vigorosa, para conseguir 10 mmol de Ti/g de arcilla. La solución se dejó reposar 30 min antes de su uso. Luego se añadió gota a gota la solución pilareante a una suspensión acuosa de $0,5 \mathrm{~mL}$ de Na-mont (1\% en peso). Después de agitar durante $24 \mathrm{~h}$, el producto sólido se centrifugó, se lavó varias veces con agua destilada, se secó al aire y luego se calcinó a $450{ }^{\circ} \mathrm{C}$ durante $3 \mathrm{~h}$. El área específica (A $\left.\mathrm{A}_{\mathrm{BET}}\right)$ de las arcillas sintetizadas fueron determinadas de las isotermas de adsorción-desorción de $\mathrm{N}_{2}$ obtenidas a $-196{ }^{\circ} \mathrm{C}$ usando un equipo Gemini V2.00 Micromeritics.

Para la transesterificación, se pesó $25 \mathrm{~g}$ de aceite de ricino en un vaso de precipitados y se calentó suavemente por 60 minutos para evaporar la humedad presente. Una masa determinada de metanol y de gránulos de $\mathrm{KOH}$, necesarios para proporcionar la relación molar deseada de metanol:aceite y la concentración de catalizador, respectivamente, se mezclaron para su disolución. El vaso de precipitados con aceite de ricino se sumergió parcialmente en un baño de agua (a una temperatura de reacción de $60{ }^{\circ} \mathrm{C}$ ) con un agitador magnético. La mezcla de metanol-KOH se introdujo en el aceite de ricino, agitando inmediatamente durante un período de tiempo. La reacción se controló de acuerdo con la siguiente variación en el tiempo de reacción 30, 45, 60, min, manteniendo constante la relación molar, la cantidad de catalizador, la temperatura de reacción y la velocidad de agitación. Después de la reacción, la mezcla se transfirió a un embudo y se dejó reposar por $24 \mathrm{~h}$ para la separación entre la fase liviana (FAME) y la fase pesada (Glicerol), ambos como productos de reacción en la transesterificación. La fase liviana se lavó con agua destilada a temperatura ambiente para eliminar trazas de jabón y/o catalizador restante. Finalmente, la mezcla se secó a $80{ }^{\circ} \mathrm{C}$ por 30 minutos en evaporador rotativo. En la Figura 3

se esquematiza el procedimiento utilizado para la Figura 3. Proceso de transesterificación. transesterificación.

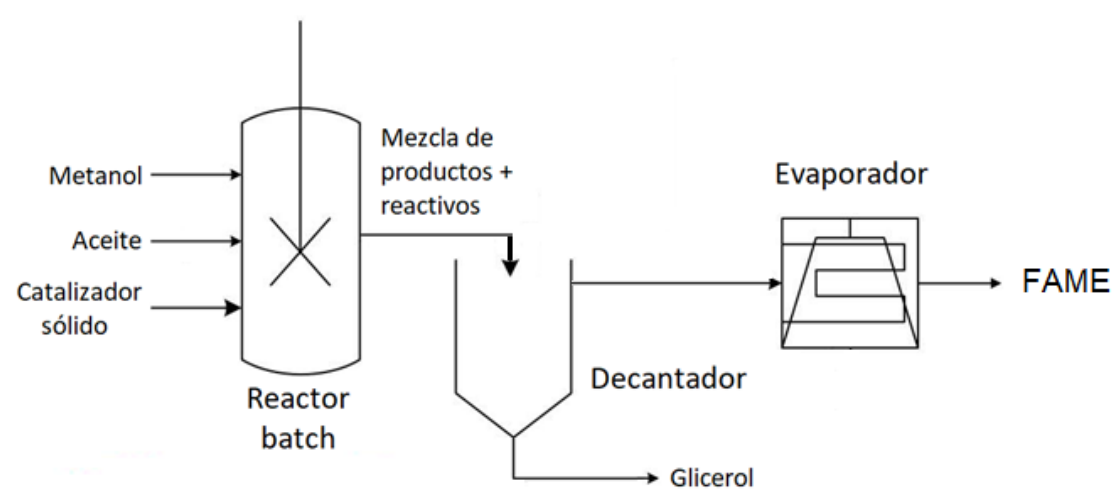

Previo a la reacción, se midió la humedad del aceite de ricino por el método Karl-Fischer, según ISO 12937:2000 y además su contenido de acidez y ácidos grasos libres (FFA). El grupo funcional principal presente en el aceite de ricino y FAME se determinó por FTIR, utilizando el equipo Thermo Scientific Nicolet iS10 con reflectancia total atenuada (ATR), en el rango de espectro entre 400-4000 $\mathrm{cm}^{-1}$ con una resolución de $4 \mathrm{~cm}^{-1}$.

\section{Resultados y Discusión}

Al caracterizar las arcillas mediante isotermas de adsorción-desorción de $\mathrm{N}_{2}$ se obtuvo un área específica de $47 \mathrm{~m}^{2} / \mathrm{g}$ y de $187 \mathrm{~m}^{2} / \mathrm{g}$ para la Na-mont y la Ti-PILC respectivamente. El material pilareado presentó área específica superior respecto a la arcilla original.

En el caso de la transesterificación, se tomaron en principio 5 variables a controlar en el proceso: la relación molar metanol: aceite, el \% en peso de catalizador, la velocidad de agitación, la temperatura y el tiempo de reacción. Para una primera aproximación se utilizó una relación molar $6: 1,0,8 \%$ en peso de $\mathrm{KOH}$ a $60^{\circ} \mathrm{C}$ durante $30 \mathrm{~min}$, con una velocidad de agitación de $350 \mathrm{rpm}$. Dicha reacción no condujo a un resultado satisfactorio debido a la formación de cantidades significativas de jabones. Debido a esto se optó por aumentar la relación a 9:1, para desplazar la reacción hacia los productos, usando 1,25\% de $\mathrm{NaOH}$ y manteniendo las demás variables iguales en la primera prueba. Nuevamente predominó la formación de jabón.

Según Deep y col. [10] varios autores han llegado a resultados inequívocos sobre la producción de FAME a partir de aceite de ricino mediante transesterificación. Estas variaciones en los resultados pueden deberse a la variación en las condiciones de la 
materia prima. Es por ello que para la siguiente prueba, previo a la reacción, se midió la cantidad de ácidos grasos libres (FFA) y el porcentaje de acidez del aceite. Cuando ambos valores superan el 1\% se debe optar por una reacción ácida (empleando $\mathrm{H}_{2} \mathrm{SO}_{4}$ como catalizador) para neutralizar los ácidos grasos libres que en grandes cantidades favorecen a la saponificación, es decir, a la formación jabones [11]. El porcentaje de FFA medido fue de 0,16\% y la acidez del 0,33 \%. Por lo cual, se descartó que la presencia de jabones sea consecuencia de la acidez del aceite. Por lo tanto, se trabajó con una relación molar mayor 12:1, para asegurarse que el exceso de metanol desplace la reacción hacia la formación de FAME desfavoreciendo la reacción secundaria (indeseada) de saponificación. Nuevamente no se obtuvieron resultados favorables.

Otra hipótesis de la formación de jabones es la cantidad de humedad que pueda tener el aceite, lo que estaría favoreciendo la saponificación. Por lo tanto, se midió la humedad del aceite por el método Karl-Fischer y se obtuvo un valor de aproximadamente $3500 \mathrm{ppm}(0,35 \%)$, el cual es considerablemente superior al recomendado $(0,06 \%)$. Con lo mencionado anteriormente, se optó previamente por secar el aceite por 60 minutos a temperaturas suaves $\left(90^{\circ} \mathrm{C}\right)$, obteniéndose así un valor de humedad de 600 ppm de agua (0,06\%). Además, se disminuyó la cantidad de catalizador a $1 \%$ en peso, ya que el aumento de $\mathrm{KOH}$ también puede favorecer la saponificación. En resumen, las condiciones operativas seleccionadas fueron: relación molar metanol:aceite $12: 1,1 \%$ de $\mathrm{KOH}$ a $350 \mathrm{rpm}, 60^{\circ} \mathrm{C}$ por 30 minutos. Con esto último se logró una mezcla, en la cual se pudo apreciar la fase liviana (FAME) y la fase pesada (Glicerol), con escaso contenido de jabón. Luego de purificar el FAME obtenido, se procedió a caracterizarlo y cuantificarlo por FTIR.

El espectro FTIR del aceite de ricino y su FAME se presenta en la Figura 4. De acuerdo con Rabelo y col. [12], la principal región espectral que permite la distinción química entre el aceite y su biodiesel resultante es $1500-1000 \mathrm{~cm}^{-1}$, que se conoce como "región de huella digital". Las bandas características del aceite se encuentran en $\sim 1097 \mathrm{~cm}^{-1}$, debido al estiramiento axial asimétrico del grupo $\mathrm{O}-\mathrm{CH}_{2}-$ $\mathrm{C}$, y a $\sim 1377 \mathrm{~cm}^{-1}$, estiramiento del grupo $\mathrm{O}-\mathrm{CH}_{2}$ correspondiente al mono, di y triglicérido. Las bandas características de FAME se ubican en $\sim 1436 \mathrm{~cm}^{-1}$, que corresponde a la flexión asimétrica de $-\mathrm{CH}_{3}$, y a $\sim 1197 \mathrm{~cm}^{-1}$ debido al estiramiento $\mathrm{O}-\mathrm{CH}_{3}$.

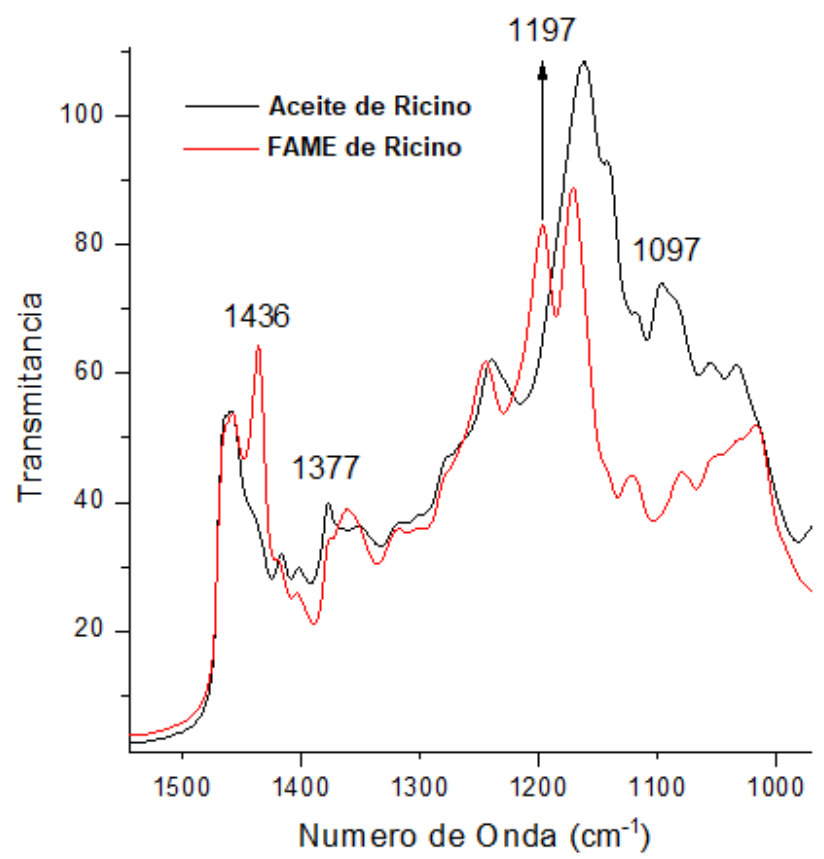

Figura 4. FTIR del aceite de ricino y su FAME resultante.

\section{Conclusiones}

Se sintetizó exitosamente la arcilla pilareada con titanio (Ti-PILC). El material pilareado presentó mayor área específica que la arcilla original, dando cuenta que las propiedades de intercambio iónico y de hinchamiento permitieron la formación de pilares. Dicho catalizador será evaluado a futuro en las reacciones de epoxidación del FAME de ricino.

En el caso de la transesterificación, se controlaron diferentes variables en el proceso: la relación molar metanol:aceite, el \% en peso de catalizador, la humedad del aceite, la velocidad de agitación, la temperatura y el tiempo de reacción. Por lo que se pudo observar, las variables más determinantes fueron la relación molar, la cantidad de catalizador y la humedad presente en el aceite. Como la velocidad de reacción viene determinada por el ataque de un nucleófilo, ya sea el ion alcóxido en la transesterificación o bien el hidróxido en la saponificación, es de suma importancia la utilización de reactivos anhidros para minimizar la presencia de agua permitiendo que el ataque del ion alcóxido sea el determinante en la velocidad de reacción, esto, sumado a un exceso de alcohol que desplaza el equilibrio hacia la formación de ésteres metílicos, permitió atenuar el efecto de 
la reacción de saponificación que no es reversible. Además, cabe mencionar, que Autores como Tomasevic y Siler-Marinkovic sugieren la utilización de $1 \%$ en peso de catalizador siempre que se mantengan constantes los demás parámetros de reacción.

Analizando los resultados obtenidos del espectro de FTIR, podemos confirmar que se ha logrado sintetizar el FAME del aceite de ricino con una conversión del $93 \%$.

\section{Referencias}

[1] Gil A., Korili S.A. y Vicente M.A. (2008). Recent Advances in the Control and Characterization of the Porous Structure of Pillared Clay Catalysts, 50, 153-221. doi: 10.1080/01614940802019

[2] Climent M.J., Corma A. e Iborra S. (2014). Conversion of biomass platform molecules into fuel additives and liquid hydrocarbon fuels. 16, 516-547. doi: 10.1039/C3GC41492B

[3] da Costa Barbosa D., Serra T., Meneghetti S.M.P. y Meneghetti M.R. (2010). Biodiesel production by ethanolysis of mixed castor oil and soybean oil. 89, 3791-3794. doi: 10.1016/j.fuel.2010.07.016

[4] Sinadinovic-Fiser S., Jankovic M. y Borota O. (2012). Epoxidation of Castor oil with peracetic acid formed in situ in the presence of an ion exchange resin. 62, 106-113. doi: 10.1016/j.cep.2012.08.005

[5] Bhuiya M.M.K., Rasul M.G., Khan M.M.K., Ashwath N. y Azad A.K. (2016). Prospects of 2nd generation biodiesel as a sustainable fuel - Part 1: Selection of feedstocks, oil extraction techniques and conversion technologies. Renewable and Sustainable Energy Reviews. 55, 1109-1128. doi: 10.1016/j.rser.2015.04.163

[6] Onukwuli D.O., Emembolu L.N., Ude C.N., Aliozo S.O. y Menkiti M.C. (2017). Optimization of biodiesel production from refined cotton seed oil and its characterization. 26, 103-110. doi: 10.1016/j.ejpe.2016.02.001

[7] Musa I.A. (2016). The effects of alcohol to oil molar ratios and the type of alcohol on biodiesel production using transesterification process. 25, 21-31. doi: 10.1016/j.ejpe.2015.06.007

[8] Elango R.K., Sathiasivan K., Muthukumaran C., Thangavelu V., Rajesh M. y Tamilarasan K. (2018). Transesterification of castor oil for biodiesel production: Process optimization and characterization. doi.org/10.1016/j.microc.2018.12.039

[9] Keera S.T., El Sabagh S.M. y Taman A.R. (2018). Castor oil biodiesel production and optimization. doi: 10.1016/j.ejpe.2018.02.007

[10] Deep A., Sandhu S.S. y Chander S. (2016). Optimization of Reaction Parameters of Transesterification for Castor Oil. ISSN: 0975-1084.

[11] Halder S., Dhawane S.H., Kumar T. y Halder G. (2015). Acid-catalyzed esterification of castor (Ricinus communis) oil: optimization through a central composite design approach. ISSN: 1759-7269. doi: 10.1080/17597269.2015.1078559

[12] Rabelo S.N., Ferraz V.P., Oliveira L.S., Franca A.S. (2015). FTIR analysis for quantification of fatty acid methyl esters in biodiesel produced by microwave -assisted transesterification. International Journal of Environmental Science and Development. 6(12), 964-969. doi: 10.7763/IJESD.2015.V6.730 